\title{
Acanthosis nigricans as a clinical marker of insulin resistance among overweight adolescents
}

\author{
Antonio Videira-Silva, MSc ${ }^{1}$, \\ Carolina Albuquerque, MD, \\ $\mathrm{MSc}^{2}$, Helena Fonseca, MD, \\ $\mathrm{MPH}, \mathrm{PhD}^{3,4}$
}

${ }^{1}$ Pediatric University Clinic, Faculty of Medicine, University of Lisbon, Lisbon, Portugal

${ }^{2}$ Department of Pediatrics, Hospital Vila Franca de Xira, Vila Franca de Xira, Portugal

${ }^{3}$ Pediatric Obesity Clinic, Department of Pediatrics, Hospital de Santa Maria, Lisbon, Portugal

${ }^{4}$ Rheumatology Research Unit, Molecular Medicine Institute, Faculty of Medicine, University of Lisbon, Lisbon, Portugal
Purpose: Acanthosis nigricans (AN) is a hyperpigmented dermatosis associated with obesity and insulin resistance (IR). There is no consensus whether AN extension scoring offers added value to the clinical estimation of IR. In this study we aimed to assess and score AN using both a short and an extended version of the scale proposed by Burke et al. and analyze the relationships of both versions with hyperinsulinemia and IR.

Methods: We analyzed data from 139 overweight adolescents (body mass index $\geq 85$ th percentile) aged $12-18$ with $(n=67)$ or without $(n=72)$ AN who were followed at a pediatric obesity clinic.

Results: Adolescents with AN had higher levels of insulin ( $d=0.56, P=0.003)$ and HOMA-IR ( $d=0.55, P=0.003$ ) compared to those without. Neither the short nor the extended versions of $A N$ scores explained either hyperinsulinemia $(\beta=1.10, P=0.316$; $\beta=1.15, P=0.251)$ or IR $(\beta=1.07, P=0.422 ; \beta=1.10, P=0.374)$. The presence of $A N$ alone predicted hyperinsulinemia and the presence of IR in $7.3 \%(\beta=2.68, P=0.008)$ and $7.1 \%(\beta=2.59, P=0.009)$ of adolescents, respectively.

Conclusion: Screening for AN at the neck and axilla is a noninvasive and costeffective way to identify asymptomatic overweight adolescents with or at risk of developing IR.

Keywords: Adolescents, Acanthosis nigricans, Overweight, Hyperinsulinemia, Insulin resistance

\section{Introduction}

Obesity is a major public health concern, associated with high cardiovascular and metabolic risks ${ }^{1,2)}$ and often leading to premature death in adulthood. ${ }^{3)}$ Among the metabolic comorbidities, hyperinsulinemia and/or insulin resistance (IR) are of particular interest due to the alarming increase of type 2 diabetes in youth. ${ }^{4)}$

Acanthosis nigricans (AN) is a dermatological condition consisting of symmetrical hyperpigmentation and thickened skin with a rough, velvety texture. AN is typically asymptomatic, although it can sometimes be pruritic. It may affect any part of the skin, but is most common in regions affected by friction. Common sites therefore include the neck, axilla, elbows, knuckles, knees, groin, and anogenital areas. ${ }^{5-7)}$ Although the pathogenesis of AN is not fully known, the underlying mechanism is the interaction between insulin-like growth factor 1 (IGF-1) and IGF binding protein 1 (IGFBP-1), which is associated with high insulin levels. Hyperinsulinemia may inhibit IGFBP-1 synthesis and increase the synthesis of IGF-1 in the liver. The increase in IGF-1 circulating concentrations and IGF-1 receptor activation may trigger dermal fibroblast and epidermal keratinocyte proliferation. ${ }^{5-8)}$

During puberty adolescents have hyperinsulinemic physiological status. ${ }^{9}$ Even higher levels of insulin are observed among overweight adolescents due to increased adipose tissue, associated with increased plasma free fatty acids and adipokines, which in turn may

https://orcid.org/0000-0002-82091676 
impair glucose transport activity, resulting in IR. IGF-1 may play an important role in this relationship. IGF-I binds to insulin receptors, stimulating glucose transport in the adipose and muscle tissues, inhibiting hepatic glucose output, and simultaneously suppressing insulin secretion, which may lead to a decrease in blood glucose. ${ }^{10)}$ However, in the presence of IR and consequent hyperinsulinemia, lower levels of IGFBP-1 may lead to disturbances in glucose homeostasis and possibly to prolonged states of IR. These factors may explain the higher prevalence of AN among overweight adolescents, compared to adults and adolescent peers who are not overweight. ${ }^{11)}$

In addition, several previous studies have reported higher AN prevalence among Black adolescents, associated with insulin hyper-secretion and IR, compared to their White peers. ${ }^{12)}$ Although it is not fully understood whether insulin hyper-secretion/IR are associated with genetic, lifestyle, and/ or socioeconomic differences, ${ }^{13)}$ there is a consensus that $\mathrm{AN}$ is associated with overweight and IR.

Although several authors have suggested that the presence of AN may be used as a clinical marker for hyperinsulinemia and IR in overweight adolescents, there is no consensus whether AN scoring adds value to the clinical estimation of IR. ${ }^{14,15)}$ Burke's scale is the most widespread measure used to score AN. ${ }^{15)}$ Two different versions of this scale (a short and an extended version) are used inconsistently across studies. In addition to this limitation, most previous studies used unbalanced group sizes for comparisons, which may have biased their conclusions.

The main aim of this study was to assess and score AN, using a short (neck and axilla) and an extended version (neck, axilla, elbows, knuckles and knees) of the scale proposed by Burke at al., ${ }^{15)}$ and to analyze the predictive power of both versions for the clinical estimation of IR.

\section{Materials and methods}

\section{Participants}

For this cross-sectional study, we analyzed data from a sample of overweight adolescents (body mass index [BMI] $\geq 85$ th percentile) aged between 12 and 18 years old who were undergoing treatment at a pediatric obesity clinic.

A total of 79 adolescents were screened for AN between July 2015 and December 2016. Of these, 67 (84.8\%) were scored according to Burke's scale. ${ }^{15)}$ Since unbalanced sample sizes can result in inaccurate statistical outcomes and may influence type I error rates ${ }^{16)}$ we added independent data for 60 adolescents who attended the clinic during the 18 -month period immediately prior to July 2015 to the without-AN group (12, corresponding to $15.2 \%$ ), for a total of 72 adolescents. Clinical data for these adolescents were collected retrospectively. The recruitment design is presented in Fig. 1.

None of the included participants met the criteria for diabetes, nor did any present with other clinical conditions or report using medication that could promote hyperinsulinemia or AN development.

\section{Measurements}

1) Anthropometric and body composition assessments

Height was assessed with a height stadiometer (seca 217, seca GmbH \& Co. KG, Hamburg, Germany) with the head in the Frankfort plane, without shoes, with the participant's back to the stadiometer, and after an expiratory phase. Height was registered to the nearest $0.1 \mathrm{~cm}$.

Body weight and body composition were measured with a bioelectrical impedance scale (InBody 230, InBody, Seoul, Korea) to the nearest $0.1 \mathrm{~kg}$, with the subjects wearing as few clothes as possible, and without shoes or socks. Relative body fat mass (\%BFM) and relative skeletal muscle mass (\%SMM) were calculated by dividing the total BFM $(\mathrm{kg})$ and SMM $(\mathrm{kg})$ by body weight, respectively.

BMI was calculated by dividing the body weight in kilograms by the square of height in meters [BMI=weight $(\mathrm{kg}) /$ height $\left.^{2}(\mathrm{~m})\right]$. The calculation of BMI $z$-score was based on World Health Organization data $[\mathrm{BMI} z$-score $=[(\mathrm{BMI} / \mathrm{M}(\mathrm{t})) \mathrm{L}(t)-1] / \mathrm{L}(t) \mathrm{S}(t)]$.

Waist (WC) and hip circumferences (HipC) were assessed using a flexible anthropometric tape (seca 203). WC was measured at the level of the iliac crest, with the subjects standing and at the end of a regular expiration (Cameron method), and HipC at the maximum protuberance of the buttocks.

\section{2) Clinical assessments}

Pubertal status was assessed and categorized according to Tanner stages. AN was assessed by the same investigator and scored according to the scale outlined by Burke et al. ${ }^{15)}$ Two different scores were calculated: AN score 1 and AN score 2. AN score 1 was calculated as the sum of the scores in each of the most prevalent AN locations (i.e., neck, axilla) and texture [AN score $1=$ neck score $(0-4)+$ axilla score $(0-4)+$ texture $(0-3)]$. AN score 2 was calculated as AN scorel, plus the presence (or absence) of AN at the elbows, knuckles, and/or knees [AN score $2=$ neck score $(0-4)+$ axilla score $(0-4)+$ texture $(0-3)+$ elbows $(0-1)+$ knuckles $(0-1)+$ knees $(0-1)]$ (Supplemental Table 1$)$.

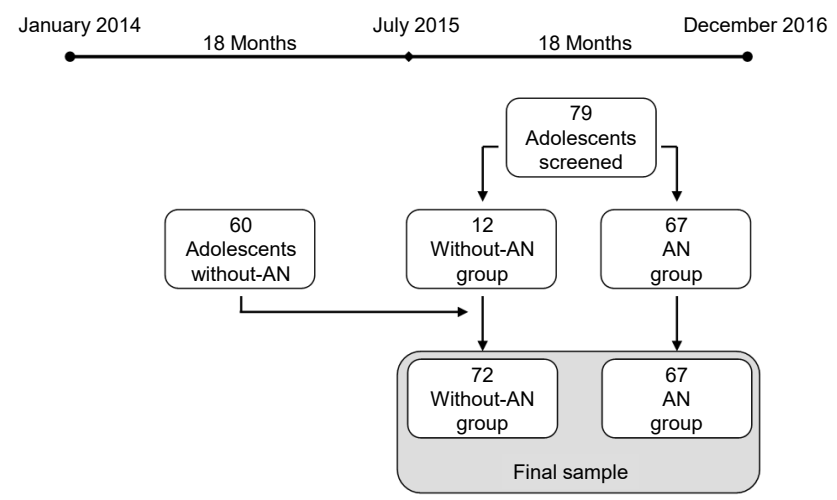

Fig. 1. Recruitment design. AN, acanthosis nigricans. 
Resting blood pressure was measured using a digital sphygmomanometer (CAS 9302S, CAS Medical Systems, Branford, CT, USA) in the right arm with an appropriate size cuff, after five minutes of rest in the seated position. The measurement was performed three times and the average of the three measurements was recorded.

Biochemical analysis was performed in the laboratory of

Table 1. Clinical characteristics of the participants

\begin{tabular}{|c|c|c|c|}
\hline Characteristic & $\begin{array}{l}\text { AN group } \\
(n=67)\end{array}$ & $\begin{array}{l}\text { Without-AN group } \\
\qquad(n=72)\end{array}$ & $P$-value \\
\hline Sex, girls & $32(47.8)$ & $31(43.1)$ & $0.578^{\mathrm{a})}$ \\
\hline Race, Black & $5(7.5)$ & $2(2.8)$ & $0.207^{\mathrm{a})}$ \\
\hline Age (mo) & $176 \pm 19$ & $170 \pm 22$ & 0.117 \\
\hline Tanner stage & & & $0.004^{a)}$ \\
\hline । & $0(0)$ & $1(1.4)$ & \\
\hline$\|$ & $1(1.5)$ & $14(19.4)$ & \\
\hline III & $12(17.9)$ & $12(16.7)$ & \\
\hline IV & $17(25.4)$ & $21(29.2)$ & \\
\hline V & $37(55.2)$ & $24(33.3)$ & \\
\hline BMI z-score & $2.51 \pm 1.34$ & $2.49 \pm 0.87$ & 0.893 \\
\hline WC (cm) & $102.4 \pm 14.2$ & $99.4 \pm 12.8$ & 0.230 \\
\hline BFM (\%) & $41.2 \pm 6.1$ & $40.3 \pm 5.6$ & 0.461 \\
\hline SMM (\%) & $32.4 \pm 6.1$ & $33.9 \pm 5.5$ & 0.129 \\
\hline $\mathrm{SBP}(\mathrm{mmHg})$ & $123.1 \pm 12.9$ & $119.0 \pm 12.6$ & 0.060 \\
\hline $\mathrm{DBP}(\mathrm{mmHg})$ & $62.8 \pm 9.5$ & $61.1 \pm 10.5$ & 0.337 \\
\hline Glycemia (mg/dL) & $84.6 \pm 8.2$ & $84.1 \pm 6.5$ & 0.693 \\
\hline Insulin $(\mu \mathrm{IU} / \mathrm{mL})$ & $26.4 \pm 16.3$ & $19.0 \pm 10.3$ & 0.003 \\
\hline HOMA-IR & $5.59 \pm 3.51$ & $4.00 \pm 2.29$ & 0.003 \\
\hline Total cholesterol (mg/dL) & $159.5 \pm 27.7$ & $152.8 \pm 28.0$ & 0.169 \\
\hline $\mathrm{HDL}-\mathrm{C}(\mathrm{mg} / \mathrm{dL})$ & $47.2 \pm 11.3$ & $50.8 \pm 12.7$ & 0.084 \\
\hline Triglycerides (mg/dL) & $94.5 \pm 55.1$ & $79.0 \pm 39.3$ & 0.064 \\
\hline $\begin{array}{l}\text { Hyperinsulinemia } \\
\text { ( } \geq 90 \text { th percentile) }\end{array}$ & $47(70.2)$ & $34(47.2)$ & $0.008^{\mathrm{a})}$ \\
\hline $\begin{array}{l}\text { IR } \\
\text { (HOMA-IR } \geq 90 \text { th percentile) }\end{array}$ & $42(62.7)$ & $28(38.9)$ & $0.008^{\mathrm{a})}$ \\
\hline AN neck & $57(85.1)$ & - & - \\
\hline AN axilla & $50(74.6)$ & - & - \\
\hline AN elbows & $26(38.8)$ & - & - \\
\hline AN knuckles & $16(23.9)$ & - & - \\
\hline AN knees & $14(20.9)$ & - & - \\
\hline
\end{tabular}

Values are presented as number (\%) or mean \pm standard deviation. BMI, body mass index; WC, waist circumference; BFM, body fat mass; SMM, skeletal muscle mass; SBP, systolic blood pressure; DBP, diastolic blood pressure; HOMA-IR, homeostatic model assessment-insulin resistance; $\mathrm{HDL}-\mathrm{C}$, high-density lipoprotein cholesterol; AN, acanthosis nigricans.

${ }^{\text {a) }}$ Chi-square test.
Clinical Pathology at the hospital. Blood samples were collected after overnight fasting (12 hours) in the presence of one of the parents/caregivers, and after local application of a topical anesthesia patch (eutectic mixture of local anesthetics, EMLA). Blood glucose levels were determined using the hexokinase method, and insulin was assessed using a chemiluminescence immunoassay technique (ADVIA 2400, Siemens, Newark, DE, USA). IR was derived by the homeostasis model assessment (HOMA-IR) method, as follows: insulin $(\mu \mathrm{UI} / \mathrm{mL}) \times$ glucose $(\mathrm{mg} / \mathrm{dL} / 405)$. Insulin and HOMA-IR values $\geq 90$ th percentile were considered to represent hyperinsulinemia and IR, respectively. ${ }^{17,18)}$ (Supplemental Table 2).

\section{Statistical analysis}

Data were analyzed using IBM SPSS Statistics ver. 22.0 (IBM Co., Armonk, NY, USA).

Chi-square tests and independent sample t-tests were used to analyze differences between the without-AN and AN groups. Logistic regression analyses were conducted to analyze the influence of AN severity scores on IR and hyperinsulinemia.

A $P$-value of $<0.05$ was considered statistically significant and odds values $>1.5$ as the minimum acceptable effect size.

\section{Results}

Data from 139 overweight adolescents (45.3\% girls, 5.2\% Black), with a mean age of $14.4( \pm 1.8)$ years and mean BMI $z$-score $2.50( \pm 1.1)$, were included in the analysis. There was no significant difference in the number of Black adolescents between the AN and without-AN groups (7.5 vs. $2.8 \%, P=0.207$ ).

The neck was the most prevalent AN location (85.1\%), followed by the axilla (74.6\%), elbows (38.8\%), knuckles (23.9\%), and knees (20.9\%) (Table 1). All participants with AN at the elbows, knuckles, and/or knees also had AN at the neck and/or axilla, but not vice-versa (data not shown).

Adolescents with AN ( $\mathrm{n}=67)$ showed higher levels of insulin $(d=0.56, P=0.003)$ and HOMA-IR $(d=0.55, P=0.003)$, and higher prevalence of hyperinsulinemia $(70.2 \%$ vs. $47.2 \%, P=0.008)$ and IR ( $62.7 \%$ vs. $38.9 \%, P=0.008)$, compared to adolescents without $\mathrm{AN}(\mathrm{n}=72)$ (Table 1). In addition, insulin and HOMAIR levels tended to be higher among adolescents with AN and IR, compared to adolescents without AN but with IR $(d=0.45$, $P=0.065 ; d=0.44, P=0.067$ ) (data not shown).

None of the AN severity scores was able to explain either hyperinsulinemia $(\beta=1.10, P=0.316 ; \beta=1.15, P=0.251)$ or IR

Table 2. Summary of logistic regression analysis for AN e AN severity scores for the prediction of hyperinsulinemia and insulin resistance

\begin{tabular}{|c|c|c|c|c|c|c|c|c|c|c|c|c|}
\hline & \multicolumn{6}{|c|}{ Hyperinsulinemia } & \multicolumn{6}{|c|}{ IR } \\
\hline & $\beta$ & SE & OR & $P$-value & Sensitivity & Specificity & $\beta$ & SE & OR & $P$-value & Sensitivity & Specificity \\
\hline AN score 1 & 0.091 & 0.091 & 1.10 & 0.316 & & & 0.065 & 0.081 & 1.07 & 0.422 & & \\
\hline AN score 2 & 0.141 & 0.123 & 1.15 & 0.251 & & & 0.097 & 1.09 & 1.10 & 0.374 & & \\
\hline AN (presence) & 0.987 & 0.374 & 2.68 & 0.008 & $66.0 \%$ & $58.0 \%$ & 0.952 & 0.362 & 2.59 & 0.009 & $63.3 \%$ & $60.0 \%$ \\
\hline
\end{tabular}

$\mathrm{AN}$, acanthosis nigricans; IR, insulin resistance; $\beta$, unstandardized beta; OR, odds ratio; SE, standard error for the unstandardized beta. 
( $\beta=1.07, P=0.422 ; \beta=1.10, P=0.374)$. However, the presence of AN alone predicted hyperinsulinemia and the presence of IR in $7.3 \%(\beta=2.68, P=0.008)$ and $7.1 \%(\beta=2.59, P=0.009)$ of subjects, respectively. Adolescents with $\mathrm{AN}$ had 2.59 and 2.68 greater odds of having hyperinsulinemia or IR, respectively, compared to those without AN (Table 2).

\section{Discussion}

A review of the literature suggests that $\mathrm{AN}$ is a clinical marker for hyperinsulinemia and IR in overweight adolescents. ${ }^{19)}$ Although one could expect that more severe AN would be associated with more severe IR, there is no consensus in this regard. ${ }^{15,20,21)}$ Results indicating the relevance of AN scoring for the prediction of IR severity may be biased by inconsistent application of the scoring system and unbalanced sample sizes. In order to overcome these limitations, we opted to use both versions of the AN scale proposed by Burke et al. ${ }^{15)}$ and to ensure balanced sample sizes.

Our findings suggest that AN scoring is not useful for the estimation of hyperinsulinemia or IR. However, the presence of $\mathrm{AN}$ alone predicted hyperinsulinemia and the presence of IR in $7.3 \%$ and $7.1 \%$ of subjects, with sensitivities of $66.0 \%$ and $63.3 \%$, respectively.

$\mathrm{AN}$ is a dermatological condition triggered by high insulin levels, usually as a consequence of IR status. ${ }^{6-8)}$ We postulate that $\mathrm{AN}$ is an ongoing process sensitive not only to insulin level variation, but also to the duration of IR status. Persistent/chronic high insulin levels may induce dermal fibroblasts and epidermal keratinocyte proliferation, AN development, and increased AN severity/extension. However, high AN severity may occur in the presence of high insulin levels during a shorter time frame of exposure.

About $63 \%$ of the adolescents in the AN group exhibited IR. It is possible that the remaining 37\% developed IR at a specific point in time, with ulterior reversion, and consequent insulin level normalization, leading to reduction of the AN extent overtime, but not to its complete reversion. ${ }^{5,6,22,23)}$ On the other hand, the $39 \%$ of adolescents without AN with IR could have developed IR more recently, and/or their insulin levels were not high enough to trigger AN development. Indeed, despite IR, adolescents without AN have lower levels of insulin, compared to those with AN.

This balance between time of exposure to high insulin levels and the severity of hyperinsulinemia may explain the non-linear relationship observed between insulin/HOMA-IR and AN severity score. In order to understand this relationship, further longitudinal studies are needed.

Although the difference was not statistically significant, it is interesting to observe that the number of Black adolescents was higher in the AN group, which is in line with the results reported by previous studies. ${ }^{12)}$ Nevertheless, because the difference in the number of Black adolescents between the AN and withoutAN groups was not statistically significant, we hypothesized that the effects on the results were also not significant.

This study has some limitations. One is the cross-sectional design, which does not allow for causal inferences. Another possible limitation is the addition of data from 60 adolescents without AN, which were retrospectively collected. Although this addition may result in bias, the reason for the use of retrospective data was to balance the sample size, to address one of the main concerns of this study. In addition, all retrospective data were collected by the same healthcare professionals.

Despite the acknowledged limitations, the results of this study suggest that the neck and axilla should be routinely assessed for the presence of $\mathrm{AN}$, since it is a noninvasive and cost-effective way to identify asymptomatic overweight adolescents who have or are at risk of developing IR, which can be associated with early development of type 2 diabetes mellitus. ${ }^{19,24)}$

Since AN may have a strong visual impact, its presence alone may be a strong reason for seeking medical care during adolescence. In addition, due to the psychological distress that it may cause, ${ }^{25)}$ AN can be used as a motivational trigger for lifestyle changes and weight loss, through the enhancement of diet and physical exercise.

\section{Ethical statement}

This study was approved by the Research Ethics Committee of the Faculty of Medicine of the University of Lisbon, Portugal $(24 / 06 / 2015)$, and is in accordance with the 1964 Helsinki declaration and its later amendments. Informed consent and assent were collected from all patients.

\section{Conflict of interest}

No potential conflict of interest relevant to this article was reported.

\section{Supplementary material}

Supplementary Table 1 and 2 can be found via http://doi. org/10.6065/apem.2019.24.2.99.

\section{Acknowledgments}

AVS is supported by the Portuguese Foundation for Science and Technology (SFRH/BD/130193/2017). This research did not receive any specific grants from funding agencies in the public, commercial, or not-for-profit sectors.

The AVS funding organization played no role in the study design; in the collection, analysis, and interpretation of data; in the writing of the report; or in the decision to submit the report for publication.

The other authors have no financial relationships relevant to this article to disclose. The authors have no potential conflicts of interest to disclose. 


\section{References}

1. Sanders RH, Han A, Baker JS, Cobley S. Childhood obesity and its physical and psychological co-morbidities: a systematic review of Australian children and adolescents. Eur J Pediatr 2015;174:715-46.

2. Reilly JJ. Obesity in childhood and adolescence: evidence based clinical and public health perspectives. Postgrad Med J 2006;82:429-37.

3. Reilly JJ, Kelly J. Long-term impact of overweight and obesity in childhood and adolescence on morbidity and premature mortality in adulthood: systematic review. Int J Obes (Lond) 2011;35:891-8.

4. Rosenbloom AL, Joe JR, Young RS, Winter WE. Emerging epidemic of type 2 diabetes in youth. Diabetes Care 1999;22:345-54.

5. Phiske MM. An approach to acanthosis nigricans. Indian Dermatol Online J 2014;5:239-49.

6. Schwartz RA. Acanthosis nigricans. J Am Acad Dermatol 1994;31:1-19.

7. Sinha S, Schwartz RA. Juvenile acanthosis nigricans. J Am Acad Dermatol 2007;57:502-8.

8. González-Saldivar G, Rodríguez-Gutiérrez R, OcampoCandiani J, González-González JG, Gómez-Flores M. Skin manifestations of insulin resistance: from a biochemical stance to a clinical diagnosis and management. Dermatol Ther (Heidelb) 2017;7:37-51.

9. Roemmich JN, Clark PA, Lusk M, Friel A, Weltman A, Epstein LH, et al. Pubertal alterations in growth and body composition. VI. Pubertal insulin resistance: relation to adiposity, body fat distribution and hormone release. Int J Obes Relat Metab Disord 2002;26:701-9.

10. Clemmons DR. Role of insulin-like growth factor iin maintaining normal glucose homeostasis. Horm Res 2004;62 Suppl 1:77-82.

11. Ng HY. Acanthosis nigricans in obese adolescents: prevalence, impact, and management challenges. Adolesc Health Med Ther 2016;8:1-10.

12. Stuart CA, Pate CJ, Peters EJ. Prevalence of acanthosis nigricans in an unselected population. Am J Med 1989;87:269-72.

13. Hannon TS, Bacha F, Lin Y, Arslanian SA. Hyperinsulinemia in African-American adolescents compared with their American white peers despite similar insulin sensitivity: a reflection of upregulated beta-cell function? Diabetes Care 2008;31:1445-7.
14. Rafalson L, Pham TH, Willi SM, Marcus M, Jessup A, Baranowski T. The association between acanthosis nigricans and dysglycemia in an ethnically diverse group of eighth grade students. Obesity (Silver Spring) 2013;21:E328-33.

15. Burke JP, Hale DE, Hazuda HP, Stern MP. A quantitative scale of acanthosis nigricans. Diabetes Care 1999;22:16559.

16. Rusticus SA, Lovato CY. Impact of sample size and variability on the power and type i error rates of equivalence tests: a simulation study. Pract Assess Res Eval 2014;19:1-10.

17. Barja S, Arnaiz P, Domínguez A, Villarroel L, Cassis B, Castillo O, et al. Normal plasma insulin and HOMA values among Chilean children and adolescents. Rev Med Chil 2011;139:1435-43.

18. García Cuartero B, García Lacalle C, Jiménez Lobo C, González Vergaz A, Calvo Rey C, Alcázar Villar MJ, et al. The HOMA and QUICKI indexes, and insulin and C-peptide levels in healthy children. Cut off points to identify metabolic syndrome in healthy children. An Pediatr (Barc) 2007;66:481-90.

19. Abraham C, Rozmus CL. Is acanthosis nigricans a reliable indicator for risk of type 2 diabetes in obese children and adolescents? A systematic review. J Sch Nurs 2012;28:195205.

20. Kobaissi HA, Weigensberg MJ, Ball GD, Cruz ML, Shaibi GQ, Goran MI. Relation between acanthosis nigricans and insulin sensitivity in overweight Hispanic children at risk for type 2 diabetes. Diabetes Care 2004;27:1412-6.

21. Koh YK, Lee JH, Kim EY, Moon KR. Acanthosis nigricans as a clinical predictor of insulin resistance in obese children. Pediatr Gastroenterol Hepatol Nutr 2016;19:251-8.

22. Kuroki R, Sadamoto Y, Imamura M, Abe Y, Higuchi K, Kato $\mathrm{K}$, et al. Acanthosis nigricans with severe obesity, insulin resistance and hypothyroidism: improvement by diet control. Dermatology 1999;198:164-6.

23. Kutlubay Z, Engin B, Bairamov O, Tüzün Y. Acanthosis nigricans: a fold (intertriginous) dermatosis. Clin Dermatol 2015;33:466-70.

24. Mizokami-Stout K, Cree-Green M, Nadeau KJ. Insulin resistance in type 2 diabetic youth. Curr Opin Endocrinol Diabetes Obes 2012;19:255-62.

25. Brickman WJ, Binns HJ, Jovanovic BD, Kolesky S, Mancini AJ, Metzger BE, et al. Acanthosis nigricans: a common finding in overweight youth. Pediatr Dermatol 2007;24:601-6. 